\title{
Antimicrobial Activity of Linezolid Against Gram-Positive Cocci Isolated in Brazil
}

\author{
Helio S. Sader, Ana C. Gales and Ronald N. Jones
}

\begin{abstract}
Special Clinical Microbiology Laboratory, Division of Infectious Diseases, Federal University of São Paulo, São Paulo, SP, Brazil; Medical Microbiology Division, Department of Pathology, University of Iowa College of Medicine, Iowa City, Iowa, USA
\end{abstract}

\begin{abstract}
The new oxazolidinone linezolid and other antimicrobial agents used to treat Gram-positive infections were tested against 1,585 Gram-positive cocci; 1,260 staphylococci and enterococci isolates from patients hospitalized in Brazilian hospitals, and $325 \mathrm{~S}$. pneumoniae isolates for patients with community acquired infections. Susceptibility testing was performed using broth microdilution according to NCCLS procedures. Linezolid was the most active compound and the only drug that inhibited $100 \%$ of the isolates at the susceptible breakpoint $(\leq 4 \mathrm{mg} / \mathrm{mL})$. Resistance to vancomycin was very rare (99.9\% susceptibility), and both quinupristin/dalfopristin and gatifloxacin were active against approximately $90 \%$ of the strains evaluated. All other compounds inhibited less than $65 \%$ of the isolates. The excellent in vitro Gram-positive activity by linezolid, in this study, indicate that this compound may represent an important therapeutic option for the treatment of infections caused by these pathogens in Brazil.

Key Words: Gram-positive cocci, oxazolidinones, antimicrobial resistance, linezolid, vancomycin resistance, nosocomial infections.
\end{abstract}

Gram-positive organisms have developed a broad range of mechanisms to evade antimicrobial agents. Of particular concern has been the emergence of glycopeptide-resistant enterococci and glycopeptideintermediate Staphylococcus aureus [1-3]. Oxacillin resistance among $S$. aureus, and penicillin resistance among Streptococcus pneumoniae and viridans group streptococci, also represent important therapeutic problems in some patients [4-6]. In addition, coagulase-

Received on 31 May 2001; revised 14 July 2001.

Address for correspondence: Prof. Hélio S. Sader, MD. Laboratório Especial de Microbiologia Clínica, Disciplina de Doenças Infecciosas e Parasitárias, Universidade Federal de São Paulo - EPM. Rua Botucatu, 740. São Paulo, SP. Zip Code: 04023-063 - Brazil. Phone: (55 11) 5081-2819/5571-5180/55764393. Fax: (55 11)5543-3013/5571-5180.

E-mail: heliosader@uol.com.br

The Brazilian Journal of Infectious Diseases 2001;5(4):171-176 (C) 2001 by The Brazilian Journal of Infectious Diseases and Contexto Publishing. All rights reserved.

$1413-8670$ negative staphylococci (CoNS) has recently become an important cause of nosocomial infections, and this pathogen has presented high rates of resistance to oxacillin and glycopeptides [7].

As a result, new antimicrobial agents are being developed, and older compounds re-evaluated, as potential alternatives for the management of infections due to multiply resistant Gram-positive microorganisms. Everninomicins [8], streptogramins [9, 10]; glycopeptide derivatives [11]; carbapenems [12]; daptomycin [13], and the oxazolidinones [14] are all in various stages of development for the treatment of Gram-positive infections.

Linezolid is a member of the oxazolidinone class of synthetic antibacterial agents that inhibit bacterial protein synthesis through a unique mechanism. In contrast to other inhibitors of protein synthesis, the oxazolidinones act early in the translation by preventing the formation of a functional initiation complex [15]. Consequently, linezolid is not expected to show cross-resistance with existing antimicrobial agents. Linezolid has remarkably 
consistent inhibitory activity against staphylococci, enterococci, and pneumococci, with MICs of 1-4 mg/ $\mathrm{mL}$ [16]. It also has moderate activity against Bacteroides spp. and Moraxella catarrhalis (MIC 8 $\mathrm{mg} / \mathrm{mL}$ ), but other Gram-negative bacteria are resistant as a result of endogenous efflux mechanisms [17].

The objective of this study was to evaluate the in vitro activity of linezolid against Gram-positive cocci isolated in Brazil in the 3-year period previous to the commercialization of the drug in this country.

\section{Materials and Methods}

The isolates were collected and tested as part of the ongoing SENTRY Antimicrobial Resistance Surveillance Program [5]. A total of 1,260 Gram-positive cocci were collected from nosocomial infections during the period of January, 1997, to December, 1999, in 4 Brazilian medical centers. In addition, 325 S. pneumoniae isolates from community acquired respiratory infections were collected and tested in the same period of time and included in the study. The participating centers included the Laboratório Especial de Microbiologia Clínica/São Paulo Hospital (LEMC - coordinator center for SENTRY Latin America), São Paulo; Laboratório Médico Santa Luzia, Florianópolis; Laboratório Lâmina, Rio de Janeiro (1997 and 1998 only); and Hospital de Clínicas de Porto Alegre, Porto Alegre (1999 only).

The isolates were consecutively collected according to the site of infection including bloodstream, lungs, wound and soft tissue, and urinary tract infections in hospitalized patients; and community acquired respiratory tract infection. Only isolates judged to be the cause of infection by local physicians were included in this study. The isolates recovered in the participant clinical microbiology laboratories were shipped to the University of Iowa College of Medicine (Iowa City, Iowa, USA), the monitoring laboratory.

Antimicrobial susceptibility testing was performed at the coordinating laboratory using broth microdilution methods as described by the National Committee for Clinical Laboratory Standards [18]. Antimicrobial agents were obtained from respective manufacturers as laboratory grade powder and included the oxazolidinone linezolid; the streptogramin quinupristindalfopristin; the glycopeptides vancomycin and teicoplanin; the b-lactams penicillin, ampicillin, and oxacillin; the fluoroquinolones ciprofloxacin and gatifloxacin; the macrolide erythromycin; the aminoglycoside gentamicin; and other compounds such as clindamycin and trimethoprim-sulfamethoxazole. The enterococci were tested against gentamicin only to detect high level resistance (MIC $>500 \mathrm{mg} / \mathrm{mL}$ ). The susceptibility breakpoint used for linezolid was $\leq 4 \mathrm{mg}$ / $\mathrm{mL}$ [14]. Breakpoints for all other compounds followed the NCCLS standards [18]. Quality control measures were utilized by testing Staphylococcus aureus ATCC 29213 and Enterococcus faecalis ATCC 29212.

\section{Results}

The Gram-positive microorganism most frequently isolated during the period of the study was $S$. aureus (852 strains, $53.8 \%$ ), followed by Streptococcus pneumoniae (325 strains, 20.5\%) coagulase-negative staphylococci (CoNS, 261 strains, 16.5\%) and Enterococcus spp. (147 strains, 9.3\%). All other Gram-positive species were isolated only occasionally and were not included in the analysis. The vast majority of $S$. pneumoniae isolates were collected from community acquired respiratory infections, while staphylococci and enterococci were collected from hospitalized patients.

Linezolid was the only compound active against $100 \%$ of the strains tested. One isolate (Enterococcus spp.) was resistant to vancomycin (99.9\% susceptibility), and 32 isolates (2.0\%) were considered non-susceptible to teicoplanin (Tables 1 and 2). The majority of isolates with non-susceptible teicoplanin MICs were CoNS (Table 1). Quinupristin/dalfopristin was very active against staphylococci and pneumococci (>99\% susceptibility), but this compound showed poor activity against Enterococcus spp. (3.4\% susceptibility) due to intrinsic resistance among $E$. faecalis.

Thirty-four percent of $S$. aureus isolates were resistant to oxacillin, and the vast majority of oxacillinresistant $S$. aureus (ORSA) isolates showed crossresistance to clindamycin, ciprofloxacin, trimethoprim/ 
sulfamethoxazole, and gentamicin. The rate of oxacillin resistance was much higher among CoNS (>80\%), and only linezolid and vancomycin were active against more than $90 \%$ of the strains evaluated.

Penicillin was active against $76.3 \%$ of $S$. pneumoniae isolates at the susceptible breakpoint (MIC $\leq 0.06 \mathrm{mg} / \mathrm{mL}$ ). The majority of penicillin nonsusceptible strains showed low level resistance (MIC $0.12-1 \mathrm{mg} / \mathrm{mL}$ ) and only $3.4 \%$ of isolates showed high level resistance (MIC $\geq 2 \mathrm{mg} / \mathrm{mL}$ ). Trimethoprimsulfamethoxazole showed poor activity against pneumococci ( $\left.\mathrm{MIC}_{90}, 4 \mathrm{mg} / \mathrm{mL}\right)$ and inhibited only $50.8 \%$ of isolates at the susceptible breakpoint.

\section{Discussion}

The emergence of antimicrobial resistance among Gram-positive species has been rapid and alarming [19]. The staphylococci have always demonstrated a remarkable ability to develop resistance to each new molecular entity of antimicrobials, and to spread effectively among patients, institutions, and communities [20]. Resistance to penicillinase-resistant penicillins (PRPs, oxacillin or methicillin) has become widespread in S. aureus and CoNS at many medical centers and in most nations [19, 21]. Staphylococci that are resistant to PRPs are frequently resistant to other antimicrobials including the cephalosporins, tetracyclines, macrolides, lincosamides, aminoglycosides, and sulfonamides. Although initially very susceptible to fluoroquinolones, oxacillin-resistant staphylococci (ORS) strains have demonstrated a rapid development of resistance to these agents, particularly when they have been used as monotherapy [22].

The glycopeptides, vancomycin or teicoplanin, remain the preferred therapy for serious infections with ORS. At the present time, high-level resistance has not been reported in a clinical isolate of $S$. aureus, although this vancomycin-resistance genome of Enterococcus has been passed into a strain of ORSA [23]. S. aureus strains with reduced susceptibility to teicoplanin have been reported regularly from Europe and the USA [19]. Resistance to teicoplanin is usually encountered among S. haemolyticus and S. epidermidis [7]. Vancomycin resistance is uncommon, but low level resistance has been recently described in isolates of $S$. aureus in the USA and Japan [1].

Vancomycin-resistant enterococci (VRE) began to be recognized in the late 1980s, and has been reported worldwide [2, 19]. Increasing prevalence of VRE have been documented among patient populations at risk, institutions, and geographic areas and very few therapeutic options remain for treatment of multiplyresistant enterococci [2, 21]. Glycopeptide resistance rates are still very low among enterococci in Brazil, and vancomycin was active against the vast majority of Gram-positive cocci evaluated in the present study (Table 2). However, several hospitals have already reported cases of VRE infections, and both intra- and inter-hospital dissemination of this pathogen has been documented in Sao Paulo [3, 10, 24]. In addition, the vast majority of VRE reported in Sao Paulo are also resistant to teicoplanin [10].

Other antimicrobial agents that could be used as empiric therapy for Gram-positive infections were evaluated. The newer quinolone, gatifloxacin, showed a similar general spectrum to that of quinupristin/ dalfopristin (Table 2). However, ORS shows some resistance to this compound, and the gatifloxacin clinical efficacy against infections caused by ciprofloxacinresistant staphylococci still must be evaluated in large clinical trials.

Another important therapeutic concern has been the increasing rates of b-lactam resistance among $S$. pneumoniae and other streptococci. Penicillin resistance remains relatively low in Brazil. However, some recent studies have shown an important increase in prevalence of penicillin-resistant strains, which usually show cross-resistance with other compounds, except the newer quinolones $[6,25]$.

As a result of the increase in antimicrobial resistance among Gram-positive bacteria, new compounds have been developed. Linezolid is one of the most promising drugs [17]. In the present study, linezolid was the only compound that showed in vitro activity against $100 \%$ of the tested organisms at the susceptible breakpoint (Tables 1 and 2). The other new compound evaluated, quinupristin/dalfopristin, had poor activity against $E$. 
Table 1. Antimicrobial activity and spectrum of linezolid and other antimicrobial agents used to treat Gram-positive infections according to the species

\begin{tabular}{|c|c|c|c|c|c|c|c|c|}
\hline \multirow[t]{3}{*}{ Antimicrobial agents } & \multicolumn{8}{|c|}{ Pathogen (no. tested) } \\
\hline & \multicolumn{2}{|c|}{ S. aureus $(\mathbf{8 5 2})$} & \multicolumn{2}{|c|}{ CoNS (261) } & \multicolumn{2}{|c|}{ Enterococcus spp. (147) } & \multicolumn{2}{|c|}{ S. pneumoniae (325) } \\
\hline & $\mathrm{MIC}_{50} / \mathrm{MIC}_{90}$ & $\%$ Susc. & $\mathrm{MIC}_{50} / \mathrm{MIC}_{90}$ & $\%$ Susc. & $\mathrm{MIC}_{50} / \mathrm{MIC}_{90}$ & $\%$ Susc. & $\mathrm{MIC}_{50} / \mathrm{MIC}_{90}$ & $\%$ Susc. \\
\hline Linezolid & $2 / 4$ & 100.0 & $1 / 2$ & 100.0 & $2 / 2$ & 100.0 & $1 / 1$ & 100.0 \\
\hline Quinupristin/dalfopristin & $0.25 / 0.5$ & 99.8 & $0.25 / 1$ & 98.9 & $8 />8$ & 3.4 & $0.5 / 0.5$ & 100.0 \\
\hline Penicillin & $16 />32$ & 9.2 & $16 />32$ & 8.1 & $2 / 16$ & 89.1 & $0.03 / 0.25$ & 76.3 \\
\hline Ampicillin & $16 />16$ & 9.9 & $16 />16$ & 12.6 & $1 / 4$ & 98.0 & NT & NT \\
\hline Oxacillin & $0.5 />8$ & 66.0 & $>8 />8$ & 19.9 & $>8 />8$ & NA & NT & NT \\
\hline Erythromycin & $1 />8$ & 45.3 & $>8 />8$ & 39.1 & $>8>8$ & 6.1 & $0.25 / 1$ & 88.0 \\
\hline Clindamycin & $0.25 />8$ & 66.5 & $>8 />8$ & 46.4 & $>8 />8$ & NA & $\leq 0.06 / 0.25$ & 95.7 \\
\hline Ciprofloxacin & $0.5 />2$ & 65.6 & $2 />2$ & 49.8 & $2 />2$ & 49.0 & $1 / 2$ & NA \\
\hline Gatifloxacin & $0.12 / 4$ & 89.3 & $0.5 / 2$ & 92.7 & $0.5 />4$ & 73.5 & $0.25 / 0.5$ & 100.0 \\
\hline $\begin{array}{l}\text { Trimethoprim/ } \\
\text { sulfamethoxazole }\end{array}$ & $\leq 0.5 />2$ & 68.0 & $2 />2$ & 40.2 & $\leq 0.5 / 2$ & 76.9 & $0.5 / 4$ & 50.8 \\
\hline Tetracycline & $\leq 4 />8$ & 62.3 & $\leq 4 />8$ & 75.1 & $>8 />8$ & 35.4 & $\leq 2 />16$ & 65.2 \\
\hline Gentamicin & $1 />16$ & 64.8 & $16 />16$ & 41.4 & $\leq 500 />1000$ & 72.8 & NT & NT \\
\hline Teicoplanin & $1 / 2$ & 99.8 & $2 / 16$ & 88.9 & $0.25 / 0.5$ & 99.3 & NT & NT \\
\hline Vancomycin & $1 / 1$ & 100.0 & $1 / 2$ & 100.0 & $1 / 2$ & 99.3 & $0.25 / 0.5$ & 100.0 \\
\hline
\end{tabular}

Abbreviations: NA: There are no breakpoints defined by NCCLS [ ]; NT: Not tested. 
Table 2. Antimicrobial activity and spectrum of linezolid and other antimicrobial agents against all Gram-positive cocci evaluated

\begin{tabular}{lccc}
\hline Antimicrobial agents & \multicolumn{3}{c}{ Gram-positive cocci (1585) } \\
\cline { 2 - 4 } & MIC $_{\mathbf{5 0}}$ (mg/mL) & MIC $_{\mathbf{9 0}}$ (mg/mL) & \% Susceptible \\
\hline Linezolid & 2 & 4 & 100.0 \\
Quinupristin/dalfopristin & 0.5 & 1 & 90.7 \\
Gatifloxacin & 0.25 & 2 & 90.6 \\
Penicillin & 8 & $>32$ & 30.1 \\
Ampicillin & 8 & $>16$ & 32.1 \\
Erythromycin & 0.5 & 16 & 49.4 \\
Tetracycline & 4 & 16 & 62.4 \\
Trimethoprim/sulfamethoxazole & 0.5 & 4 & 60.7 \\
Vancomycin & 1 & 2 & 99.9 \\
\hline
\end{tabular}

faecalis, which is the most frequently isolated Enterococcus species in Brazilian hospitals. In addition, resistance to quinupristin/dalfopristin has been demonstrated among E. faecium isolated in Brazilian hospitals [3, 10].

In addition to the excellent in vitro Grampositive activity reported in this study and in several other investigations, intravenous and oral linezolid has produced high rates of clinical success in clinical trials involving hospitalized patients with skin or soft tissue and pneumonia [26]. Preliminary clinical data also indicate that twice daily intravenous or oral linezolid $600 \mathrm{mg}$ is as effective as intravenous vancomycin in the treatment of patients with hospital-acquired pneumonia and in those with infections caused by ORS. Linezolid dosing at 600 mg twice daily produced $>85 \%$ clinical/ microbiological cure in VRE infections [26]. Nevertheless, more data are needed on efficacy in immunosuppressed patients and for other conditions, notably endocarditis, where bactericidal activity may be necessary.

The results of our study, coupled with the results on clinical efficacy demonstrated in other studies [17, 26], indicate that linezolid may represent an excellent therapeutic option to treat infections due to Grampositive cocci in Brazil; in particular those caused by multiresistant strains.

\section{References}

1. Centers for Disease Control and Prevention. Update: Staphylococcus aureus with reduced susceptibility to vancomycin - United States, 1997. Morbid Mortal Weekly Rep 1997;46:813-5.

2. Jones R.N., Sader H.S., Erwin M.E., The Enterococcus Study Group. Emerging multiply resistant enterococci (MRE) among clinical isolates: prevalence data from 97 medical centers. Diag Microbiol Infect Dis 1995;21:8593.

3. Sader H.S., Cereda R.F., Gales A.C., et al. Molecular typing and antimicrobial susceptibillity of vancomycinresistant enterococci (VRE) in Brazil. In: Abstracts of the $9^{\text {th }}$ International Congress on Infectious Diseases, Buenos Aires, Argentina, April 9 - 13, 2000. Abstract 51.006.

4. Bochud P., Calandra T., Francioli P. Bacteremia due to viridans streptococci in neutropenic patients: a review. Am J Med 1994;97:256-64.

5. Sader H.S., Sampaio J.L.M., Zoccoli C., Jones R.N. Results of the SENTRY antimicrobial surveillance program results in three Brazilian medical centers for 1997. Braz J Infect Dis 1999;3:63-79. 
6. Sader H.S., Gales A.C., Granacher T.L., et al. Prevalence of antimicrobial resistance among respiratory tract isolates in Latin America: Results from SENTRY Antimicrobial Surveillance Program (1997-98). Braz J Infect Dis 2000;4:246-55.

7. Del' Alamo L., Sampaio J., Miranda E.A., Sader H.S. Comparative in vitro activities of moxifloxacin (BAY 12-8039) and other antimicrobial agents against respiratory tract pathogens in Brazil. Braz J Infect Dis 1999;3:215-9.

8. Jones R.N., Barrett M.S. Antimicrobial activity of SCH 27899, an oligosaccharide member of the everninomycin class with a wide Gram-positive spectrum. Clin Microbiol Infect 1995; 1 :35-43.

9. Linden P.K., Pasculle A.W., McDevitt D., et al. Effect of quinupristin-dalfopristin of the outcome of vancomycin-resistant Enterococcus faecium bacteremia: Comparison with a control cohort. J Antimicrob Chemother 1997;39(suppl A):145-51.

10. Sader H.S., Jones R.N., Ballow C.H., et al. Antimicrobial susceptibility of quinupristin/dalfopristin tested against Gram-positive cocci from Latin America: Results from the Global SMART (GSMART) Surveillance Study. Braz J Infect Dis 2001;5:21-30.

11. Jones R.N., Barrett M.S. Erwin M.E. In vitro activity and spectrum of LY333326, a novel glycopeptide derivative. Antimicrob Agents Chemother 1997;41:488-91.

12. Sader H.S., Gales A.C. Emerging strategies in infectious diseases: New carbapenem and trinem antimicrobial agents. Drugs 2001;61:553-64.

13. Alborn W.E., Allen N.E., Preston D.A. Daptomycin disrupts membrane potential in growing Staphylococcus aureus. Antimicrob Agents Chemother 1991;35:2282-7.

14. Diekema D.J., Jones R.N. Oxazolidinones: A review. Drugs 2000;59:7-16.

15. Shinabarger D.L., Marotti K.R., Murray R.W., et al. Mechanism of action of the oxazolidinones: effects of linezolid and esperezolid on translation reactions. Antimicrob Agents Chemother 1997;41:2132-6.

16. Johnson A.P., Warner M., Livermore D.M. Activity of linezolid against multi-resistant Gram-positive bacteria from diverse hospitals in the United Kingdom. J Antimicrob Chemother 2000;45:225-30.
17. Livermore D.M. Quinupristin/dalfopristin and linezolid: where, when, which and whether to use? J Antimicrob Chemother 2000;46:347-50.

18. National Committee for Clinical Laboratory Standards. Methods for dilution antimicrobial susceptibility tests for bacteria that grow aerobically; approved standard fifth edition. Approved document M7-A5. Wayne, Pennsylvania. NCCLS 2000.

19. Cormican M.G., Jones R.N. Emerging resistance to antimicrobial agents in Gram-positive bacteria. Drugs 1996;51(suppl 1)6-12.

20. Sader H.S., Pignatari A.C., Hollis R.J., Jones R.N. Evaluation of inter-hospital spread of methicillinresistant Staphylococcus aureus in Sao Paulo, Brazil, using pulsed-field gel electrophoresis of chromosomal DNA. Infect Control Hosp Epidemiol 1994;15:320-3.

21. Pfaller M.A., Jones R.N., Doern G.V., et al. Survey of blood stream infections attributable to Gram-positive cocci: frequency of occurrence and antimicrobial susceptibility of isolates collected in 1997 in the United States, Canada, and Latin America from the SENTRY Antimicrobial Surveillance Program. Diagn Microbiol Infect Dis 1999;33:283-97.

22. Ena J., Houston A., Wenzel R.P., Jones R.N. Trends in Gram-positive blood stream organism resistance: A seven-year audit of five glycopeptides and other drugs at a large university hospital. J Chemother 1993;5:17-21.

23. Noble W.C., Virani Z., Cree R.G.A. Co-transfer of vancomycin and other resistance genes from Enterococcus faecalis NTCC 12201 to Staphylococcus aureus. FEMS Microbiol Lett 1992;93:195-8.

24. Cereda R.F., Sader H.S., Jones R.N., et al. An Enterococcus faecalis strain resistant to vancomycin and teicoplanin (VanA Phenotype) isolated in a bone marrow transplanted patient in Brazil. Braz J Infect Dis 2001;5:40-6.

25. Brandileone M.C., Di Fabio J.L., Vieira V.S.D., et al. Geographic distribution of penicillin resistance of Streptococcus pneumoniae in Brazil: Genetic relatedness. Microbial Drug Resist 1998;4:209-17.

26. Clement D., Markham A. Linezolid. Drugs 2000;59:815-27. 